\title{
The role of higher-order modes on the electromagnetic whistler-cyclotron wave fluctuations of thermal and non-thermal plasmas
}

\author{
Adolfo F. Viñas, ${ }^{1}$ Pablo S. Moya, ${ }^{1,2}$ Roberto Navarro, ${ }^{3}$ and Jaime A. Araneda ${ }^{4}$ \\ ${ }^{1}$ NASA Goddard Space Flight Center, Heliophysics Science Division, Geospace Physics Laboratory, \\ Mail Code 673, Greenbelt, Maryland 20771, USA \\ ${ }^{2}$ Department of Physics, Catholic University of America, Washington DC, District of Columbia 20064, USA \\ ${ }^{3}$ Departamento de Física, Facultad de Ciencias, Universidad de Chile, Casilla 653, Santiago, Chile \\ ${ }^{4}$ Departamento de Física, Universidad de Concepción Facultad de Ciencias Físicas y Matemáticas, \\ Casilla 160-C, Concepción, Chile
}

(Received 28 October 2013; accepted 20 December 2013; published online 13 January 2014)

Two fundamental challenging problems of laboratory and astrophysical plasmas are the understanding of the relaxation of a collisionless plasmas with nearly isotropic velocity distribution functions and the resultant state of nearly equipartition energy density with electromagnetic plasma turbulence. Here, we present the results of a study which shows the role that higher-order-modes play in limiting the electromagnetic whistler-like fluctuations in a thermal and non-thermal plasma. Our main results show that for a thermal plasma the magnetic fluctuations are confined by regions that are bounded by the least-damped higher order modes. We further show that the zone where the whistler-cyclotron normal modes merges the electromagnetic fluctuations shifts to longer wavelengths as the $\beta_{e}$ increases. This merging zone has been interpreted as the beginning of the region where the whistler-cyclotron waves losses their identity and become heavily damped while merging with the fluctuations. Our results further indicate that in the case of nonthermal plasmas, the higher-order modes do not confine the fluctuations due to the effective higher-temperature effects and the excess of suprathermal plasma particles. The analysis presented here considers the second-order theory of fluctuations and the dispersion relation of weakly transverse fluctuations, with wave vectors parallel to the uniform background magnetic field, in a finite temperature isotropic bi-Maxwellian and Tsallis-kappa-like magnetized electron-proton plasma. Our results indicate that the spontaneously emitted electromagnetic fluctuations are in fact enhanced over these quasi modes suggesting that such modes play an important role in the emission and absorption of electromagnetic fluctuations in thermal or quasi-thermal plasmas. (C) 2014 AIP Publishing LLC. [http://dx.doi.org/10.1063/1.4861865]

\section{INTRODUCTION}

The processes of kinetic plasma relaxation and turbulence generation are responsible for many of the observed properties of laboratory and astrophysical plasmas. In particular, these processes play an important role in the solar wind plasma which is the only collisionless plasma accessible to detailed in-situ satellite observations. ${ }^{1-3}$ The morphology of the plasma velocity distribution function (VDF) is generally determined by the global structure of the magnetic and electric fields, the nature of the scattering processes (e.g., Coulomb collisions, wave-particle interactions) between the source and the measurement location and the boundary conditions from which the particles emanate. Detailed measurements of the VDFs can then provide information about the propagation conditions and to some extent about the conditions in the source region itself.

In spite of the absence of free-energy for plasma instabilities, a finite-temperature plasma sustains a small but detectable spontaneous fluctuations level of absorption and emission of various collective modes of oscillations, which arises from the discreteness of the plasma particles, in other words, from the charge and current fluctuations in the plasma. In linear and spatially homogeneous plasmas, these spontaneous emissions ${ }^{4-9}$ are intimately linked to electric and magnetic field fluctuations by the well-known fluctuation-dissipation theorem. ${ }^{12}$ Spectral properties of the scattered fluctuating emission can provide substantial information about the state of a plasma. The analysis of electromagnetic wave scattering is, in fact, one of the most efficient methods of plasma diagnostics used in laboratory fusion research devices and in space-plasma measurement (e.g., Refs. 13-15).

The spontaneous thermal emissions of a particular eigenmode of a plasma serve as a seed perturbation to drive plasma instabilities of the corresponding wave modes whenever some free-energy process becomes available. The study of these fluctuations leads also to the concept of correlation functions (particularly in time) which play an important role in relating the dissipative properties of a system, such as the viscous resistance of a fluid or the electrical resistant of a conductor, with the microscopic properties of the system in a state of near-equilibrium. ${ }^{16} \mathrm{~A}$ good example of the fluctuation-dissipation theorem is the quasi-thermal noise in space plasmas which is directly linked to fluctuations of charged-particle densities and electromagnetic fields, giving rise to scattered radiation. For systems in nearly thermal equilibrium, the emitted fluctuating radiation takes on the 
spectral form of black-body radiation. Because of fluctuations of charge, current, and electromagnetic fields, the particles can be excited, for example, by Langmuir, whistlercyclotron (wc), or Alfvén waves, leading to a system that cannot be in thermal equilibrium, so that the emitted radiation deviates from the black-body approximation. However, in most cases, the state of the system can be described by the linear response to the perturbations from the equilibrium state via the fluctuation-dissipation theorem.

The most striking feature of this theorem is in a fundamental manner, which it relates the fluctuations of a physical quantity pertaining to the equilibrium state of a given system to a dissipative irreversible process, which in practice is realized only when the system is subject to an external perturbation that drives it away from its state of equilibrium. Consequently, this theorem enables us to determine the nearequilibrium properties of a given statistical system on the basis of the knowledge of the thermal fluctuations occurring in the system when the system is in one of its equilibrium states. Thus, the statistical mechanics of irreversible processes is, in a sense, reduced to the statistical mechanics of near-equilibrium states (provided we are capable of handling the time dependent fluctuation processes occurring in the system). The fundamental theory of fluctuations was advanced by Callen and Welton ${ }^{12}$ and later improved and refined by Sitenko ${ }^{17}$ which provided a detailed account of the theory. Although the fundamentals ideas of the theory of electromagnetic fluctuations have already been developed in these references (Callen and Welton ${ }^{12}$ and Sitenko ${ }^{17}$ ), the actual application of the formulation to the problem of spontaneous emission of whistler-cyclotron waves and its connection to the generally ignored higher-order modes (hom) have received little attention.

The electromagnetic dispersion relation of whistlercyclotron waves and ubiquitous higher-order modes in a thermal plasma has been previously investigated by Gitomer ${ }^{18}$ and Gitomer and Forslund ${ }^{19}$ in a study of collisionless cyclotron damping of these wave modes, using a numerical linear dispersion analysis and non-linear particle simulations in an attempt to explain electron heating via these wave eigenmodes. Murata et al. ${ }^{20}$ also investigated similar higher-order modes but associated with electrostatic ion-cyclotron waves. However, it has not been until recently that these higherorder modes have received more attention, in particular, with regards to their role in the spontaneous emission of electromagnetic fluctuations in a plasma. ${ }^{7}$

In this paper, we examine the kinetic theory of electromagnetic fluctuations associated with the electron whistlercyclotron waves in plasmas following the theoretical approach of Sitenko. ${ }^{17}$ In Sec. II, we cover the linear dispersion analysis for parallel fluctuating whistler-cyclotron waves including the dispersion characteristics of the higherorder modes in a stable-equilibrium plasma. This section gives a brief description of the complete set of whistler wave modes at parallel propagation, with particular attention paid to the topology of the often ignored higher-order modes on the dispersion analysis. In Sec. III, we discuss the general second-order nonlinear theory of fluctuations focusing our attention on the emission or absorption of electromagnetic fluctuations and their relation to the higher-order modes. The general discussion is carried out for two model distribution function: (a) bi-Maxwellian and (b) Tsallis-kappa-like VDFs. $^{21-23}$ In Sec. IV, we complemented the analytical and numerical approaches with a full particle simulation study in the stable regime of a plasma under different thermal conditions. Finally, a discussion of the results and the implication to the limiting role of the higher-order modes on the fluctuations of solar wind plasma observations is presented in Sec. V.

\section{LINEAR KINETIC THEORY AND HIGHER-ORDER MODES}

Whistler-cyclotron waves are fundamental transverse electromagnetic normal modes of magnetized plasmas with frequencies between the ion and electron gyrofrequencies, $\left|\Omega_{p}\right|<\omega<\left|\Omega_{e}\right|$. They are characterized by a helical righthanded polarization, which makes them prone to resonate with energetic electrons via cyclotron resonance. For that reason, they have traditionally received much attention in space plasma physics. These wave modes can be driven by charged particle thermal anisotropies or by differential streaming among various electron populations. For a proper understanding of the linear and nonlinear instabilities involving such waves, it is essential to understand their mode structure (both normal as well as their higher-order modes) to develop the ability to recognize them in laboratory and space observations. Higher-order modes have been studied for electrostatic waves by Derfler, ${ }^{24}$ Murata, ${ }^{20}$ and Gary and Tokar, ${ }^{25}$ and for electromagnetic waves by Gitomer, ${ }^{19}$ Matsuda, ${ }^{26}$ and Astudillo. ${ }^{27}$

In this section, we describe the linear Vlasov-Maxwell theory $^{28,29}$ to determine the full set of electron wave modes and higher-order modes for a homogeneous and magnetized plasma. We assume electromagnetic fluctuations propagating either parallel or anti-parallel to the ambient magnetic field $\mathbf{B}_{0}$. The linearized dispersion relation tensor $\Lambda_{i j}(\omega, k)$ is obtained from the Fourier-Laplace transformed VlasovMaxwell equations yielding

$$
\Lambda_{i j}(\omega, \mathbf{k})=\varepsilon_{i j}(\omega, \mathbf{k})-\frac{c^{2} k^{2}}{\omega^{2}}\left(\delta_{i j}-\frac{k_{i} k_{j}}{k^{2}}\right) .
$$

The electromagnetic plasma medium properties are completely described in terms of the plasma dielectric permittivity tensor $\varepsilon_{i j}$, which depends on the complex frequency $\omega$ and the wave-vector $\mathbf{k}$. The dispersion relation that determines the frequencies $\omega=\omega(\mathbf{k})$ is given from the condition that $\operatorname{det}(\Lambda)=0$. For propagation parallel to $\mathbf{B}_{0}$, we obtain $\operatorname{det}(\Lambda)=\Lambda_{+} \Lambda_{-} \Lambda_{\|}=0$, so that the dispersion relation for longitudinal and transverse waves are $\Lambda_{\|}=0$ and $\Lambda_{ \pm}=0$, respectively.

The transverse contributions are given by

$$
\Lambda_{ \pm}\left(\omega, k_{\|}\right)=1-\frac{c^{2} k_{\|}^{2}}{\omega^{2}}+\frac{1}{2} \sum_{s} \frac{\omega_{p s}^{2}}{\omega^{2}} \int d^{3} v \frac{v_{\perp} \chi_{s}\left(\omega, k_{\|}\right)}{\omega-k_{\|} v_{\|} \pm \Omega_{s}}
$$

and the longitudinal contribution by 


$$
\Lambda_{\|}\left(\omega, k_{\|}\right)=1+\sum_{s} \frac{\omega_{p s}^{2}}{\omega} \int d^{3} v \frac{v_{\|}}{\omega-k_{\|} v_{\|}} \frac{\partial F_{s}}{\partial v_{\|}},
$$

where we have defined

$$
\chi_{s}\left(\omega, k_{\|}\right)=\left(\omega-k_{\|} v_{\|}\right) \frac{\partial F_{s}}{\partial v_{\perp}}+k_{\|} v_{\perp} \frac{\partial F_{s}}{\partial v_{\|}} .
$$

Here, $\omega_{p s}^{2}=4 \pi n_{s} q_{s}^{2} / m_{s}$ is the squared plasma frequency and $\Omega_{s}=q_{s} B_{0} /\left(m_{s} c\right)$ is the cyclotron frequency, with $n_{s}, q_{s}$, and $m_{s}$ the density, charge, and mass of the species $s$, respectively, and $c$ is the speed of light.

For a plasma composed of parallel drifting electrons and protons that obey the bi-Maxwellian particle velocity distribution function

$$
F_{s}\left(v_{\|}, v_{\perp}\right)=\frac{1}{\pi^{3 / 2} \alpha_{\| s} \alpha_{\perp s}^{2}} \exp \left[-\frac{\left(v_{\|}-U_{\| s}\right)^{2}}{\alpha_{\| s}^{2}}-\frac{v_{\perp}^{2}}{\alpha_{\perp s}^{2}}\right],
$$

the resultant transverse electromagnetic dispersion relation becomes

$$
\begin{aligned}
\Lambda_{ \pm}\left(\omega, k_{\|}\right)= & 1-\frac{k_{\|}^{2} c^{2}}{\omega^{2}}+\sum_{s=p, e} \frac{\omega_{p s}^{2}}{\omega^{2}} \\
& \times\left[\xi_{s} Z\left(\xi_{s}^{ \pm}\right)+\Gamma_{s}\left(1+\xi_{s}^{ \pm} Z\left(\xi_{s}^{ \pm}\right)\right)\right], \\
\xi_{s}= & \frac{\omega-k_{\|} U_{\| s}}{k_{\|} \alpha_{\| s}}, \quad \xi_{s}^{ \pm}=\xi_{s} \pm \frac{\Omega_{s}}{k_{\|} \alpha_{\| s}}, \\
\Gamma_{s}= & \frac{\alpha_{\perp s}^{2}}{\alpha_{\| s}^{2}}-1=\frac{T_{\perp s}}{T_{\| s}}-1,
\end{aligned}
$$

and the resultant longitudinal electrostatic dispersion relation gives

$$
\Lambda_{\|}\left(\omega, k_{\|}\right)=1-\sum_{s=p, e} \frac{\omega_{p s}^{2}}{k_{\|}^{2} \alpha_{\| s}^{2}} Z^{\prime}\left(\xi_{s}\right)
$$

where $\Gamma_{s}$ is a measure of the temperature anisotropy, $\alpha_{\| s}=\sqrt{2 \kappa_{B} T_{|| s} / m_{s}}$ and $\alpha_{\perp s}=\sqrt{2 \kappa_{B} T_{\perp s} / m_{s}}$ are the parallel and perpendicular thermal velocities, respectively. $Z(\xi)$ and $Z^{\prime}(\xi)$ are the traditional Fried-Conte plasma dispersion function and its derivative with respect to $\xi$, respectively. ${ }^{30}$

It is well known that linear instabilities can be studied in great detail from the solutions of the electromagnetic kinetic dispersion relation without any restriction on wavelength, growth rate, direction of propagation, etc. Many characteristics of a given mode (e.g., growth or damping rate, polarization, wave vector, compressibility, etc.) can be determined by solving the dispersion equation (analytically or numerically). However, linear dispersion relation studies traditionally do not fully take into consideration the topology of the whole $\omega-k$ space. In general, only the fastest growing wave and the least damped wave modes are studied by following the solutions of the dispersion relation in the complex $-\omega$ plane for a set of fixed macroscopic parameters. Nonetheless, any kinetic dispersion relation that includes the Fried-Conte plasma dispersion function ${ }^{30}$ contains an infinite number of root-solutions that are associated to higher-order modes. These higher-order modes are also solutions of the dispersion relation. In the past, these roots have been considered unimportant because they are heavily damped. As a result, there exists a common interpretation that such heavily damped modes do not contribute significantly to the propagation characteristics of the plasma or that these modes are not physically realizable in a physical system. In this paper, however, we show that this interpretation is misleading. To investigate the nature of these higher-order modes, we numerically solved the transverse dispersion relation given in Eq. (6). We consider here the role played by these higher-order modes and their manifestation in an electron-proton plasma.

The solutions of the transverse dispersion relation $\Lambda_{ \pm}=0$ are obtained numerically for various plasma conditions and for the frequency range of interest (i.e., $\left|\Omega_{p}\right| \leq \omega$ $\leq\left|\Omega_{e}\right|$ ) by considering a non-drifting (i.e., $U_{\| s}=0$ ) isotropic plasma. For the frequency range of interest, the dynamics of the ions plays little role since they are more massive than electrons and as such high frequencies they can almost be considered unmagnetized. In Fig. 1, we show the numerical results of the transverse dispersion relation $\left(\Lambda_{ \pm}=0\right)$ for a warm plasma composed of isotropic bi-Maxwellian ions and electrons $\left(T_{\perp \mathrm{s}} / T_{\| \mathrm{s}}=1\right)$, with $\beta_{e}=\beta_{p}=0.01$ and $\omega_{p e} / \Omega_{e}=1$. Here, we show all fourquadrants for the wave vector range of $-3 \leq k_{\|} \lambda_{e} \leq 3$ and the normalized frequency $-3 \leq \operatorname{Re}(\omega) /\left|\Omega_{\mathrm{e}}\right| \leq 3$, where $\lambda_{e}=c / \omega_{p e}$ is the electron inertial length but only for the positive helicity case (i.e., the positive sign selection in Eq. (6). In the top panel [Fig. 1(a)], the solid curves crossing
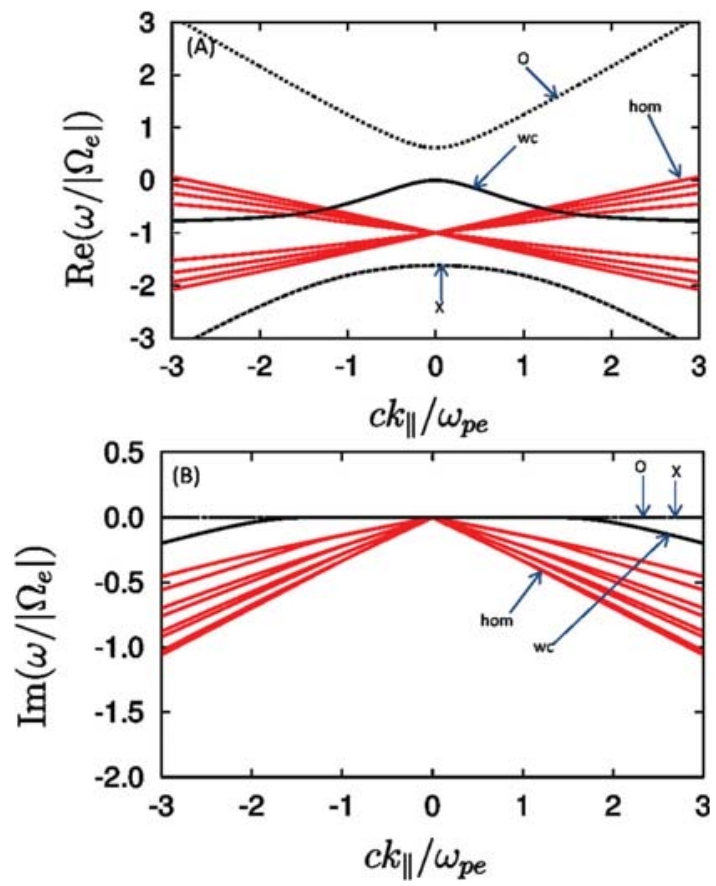

FIG. 1. (a) Real part of the solution of the transverse dispersion relation $\Lambda_{ \pm}$ $=0$ for an isotropic Maxwellian electron-proton plasma, with $m_{p} / m_{e}=1836$, $\omega_{p e} / \Omega_{e}=1$, and $\beta_{e}=0.01$. Solid curves correspond to whistler (wc), ordinary $(\mathrm{O})$ and extraordinary $(\mathrm{X})$ normal modes, and dotted lines to hom. (b) Corresponding imaginary part of the wave modes shown in panel (a). 
the origin of the $(\operatorname{Re}(\omega), k)$-plane represent the standard forward (third quadrants) and backward (fourth quadrants) propagating right-handed wc modes which approaches resonance at the electron cyclotron frequency $-\left|\Omega_{e}\right|$. There are two other wave modes corresponding to the left-handed ordinary $(\mathrm{O})$ and the right-handed extraordinary $(\mathrm{X})$ wave modes. Both modes approach asymptotically to the speed of light at higher $k_{\|}$-values. The ordinary left-handed wave modes are the dashed curves that hit the cut-off frequency at $k_{\|} \lambda_{e}=0$ just below the electron cyclotron frequency $\left(\omega \lesssim+\left|\Omega_{e}\right|\right)$, whereas the extraordinary righthanded waves are the dashed black curves that hit the cut-off frequency at $k_{\|} \lambda_{e}=0$ above the electron cyclotron frequency $\left(\omega \gtrsim-\left|\Omega_{e}\right|\right)$. These waves have their corresponding forward and backward propagation counterparts as shown on the other quadrants. Fig. 1(a) also includes eight more solutions of the dispersion relation, of an infinite set of mode solutions, corresponding to the least damped hom crossing at $\operatorname{Re}\left(\omega / \Omega_{\mathrm{e}}\right)=-1$ at $k_{\|} \lambda_{e}=0$ [red solid lines in Fig. 1(a)]. These modes has been studied before and they corresponds to the $A_{n}$ (upper) and $B_{n}$ (lower) higher-order modes of Gitomer and Forslund ${ }^{19}$ and Murata et al. ${ }^{20}$ Fig. 1(b) shows the corresponding damping rate of the main branches of the whistler-cyclotron solutions and also of the higher-order modes. Recently, Felten et al. ${ }^{9}$ have investigated a kind of aperiodic quasi-modes in an unmagnetized relativistic plasma that appear to have similarities with the higher-order modes cited here. However, the condition $\operatorname{Re}(\omega) \ll \operatorname{Im}(\omega)$ required by these aperiodic quasi-modes is not always true when compared with the higher-order modes, since they also depend on $k_{\|}$. Nonetheless, some of the higher-order modes do satisfy that condition and they could be the aperiodic quasi-modes referred by Felten et al. ${ }^{9}$ There have been further attempts to solve the dispersion relation to obtain approximate analytical solutions to the normal modes for the case of low-frequency electromagnetic Alfven waves. ${ }^{10,11}$ Such approximations for the higher-order modes have not yet been attempted, and here we limit ourselves to the numerical solution of all these modes.

Fig. 1(b) shows that for the ordinary and extraordinary modes, the damping rate is essentially zero, whereas for the whistler-cyclotron branch the damping is nearly zero at long wavelengths and then increases substantially as the wave vector increases. The damping of the higher-order modes increases in the directions given by the red solid lines in Fig. 1(b). These modes fill the $(\operatorname{Re}(\omega), k)$-plane so that the least damped higher-order modes form triangle-like zones in the dispersion diagram [Fig. 1(a)], with corners at the frequencies near 0 and $-2 \Omega_{e}$ at $k_{\|} \lambda_{e}= \pm 3$. These zones also contain portions of the whistler branches dispersion (in the fourth and second quadrant). As a consequence, we could expect that if free energy is available for the excitation of whistler-cyclotron waves, they should emerge in those regions bounded by the higher-order modes. In fact, linear Vlasov analysis predicts that the whistler-cyclotron branch can be unstable with an anisotropic bi-Maxwellian electron distribution. As an example, for a temperature ratio $T_{\perp e} / T_{\| e}=6$ and the same $\beta_{e}$ as in Fig. $1\left(\beta_{e}=0.01\right)$, the growth rate is positive in the normalized wave vector range $1.3<k_{\|} \lambda_{e}<2.4$, reaching its maximum at $k_{\|} \lambda_{e} \approx \pm 1.77$ with $\operatorname{Re}\left(\omega_{\max } /\left|\Omega_{\mathrm{e}}\right|\right) \approx-0.03$. Although not illustrated here, the increase of the temperature anisotropy makes no qualitative changes in the real part of the dispersion diagram.

Similar wave mode calculations were carried out for larger values of $\beta_{e}$. Figs. 2 and 3 illustrate the solutions of $\Lambda_{ \pm}=0$ for $\beta_{e}=0.1$ and $\beta_{e}=0.3$, respectively. Note that the zones of higher-order damped modes broaden (see Figs. 2(a) and 3(a)) with increasing $\beta_{e}$, whereas the damping rate zones decreases (see Figs. 2(b) and 3(b)). Simultaneously, the intersection between the least damped mode and the whistler-cyclotron mode shifts towards smaller values of $k_{\|} \lambda_{e}$. This result strongly suggests that with increasing $\beta_{e}$, whistler wave-particle scattering will progressively tend to occupy the lower part of the $k$-spectrum. As $T_{\perp e} / T_{\| e}$ increases, the whistler branch becomes unstable (not shown here) with the positive growth region shifted to smaller wave vectors, but still constrained by the least damped higher-order modes at small values of $k_{\|} \lambda_{e}$, and cyclotron damping at larger wave vectors. As in the previous case, the dispersion diagrams show no qualitative differences between the electron isotropy and moderate unstable cases $\left(T_{\perp e} / T_{\| e}<3\right)$. In Sec. III, we will discuss an additional kinetic effect that is closely related to the behavior of the higher-order modes and the triangle-like zones. The solutions presented in Figs. 1 to 3 have a mirror image counterparts, when the negative helicity (i.e., negative sign) is used in the dispersion relation of Eq. (6).

\section{ELECTROMAGNETIC SPONTANEOUS FLUCTUATIONS IN A PLASMA}

The knowledge of the fluctuation spectrum is the starting point for the computation of the emission of transverse
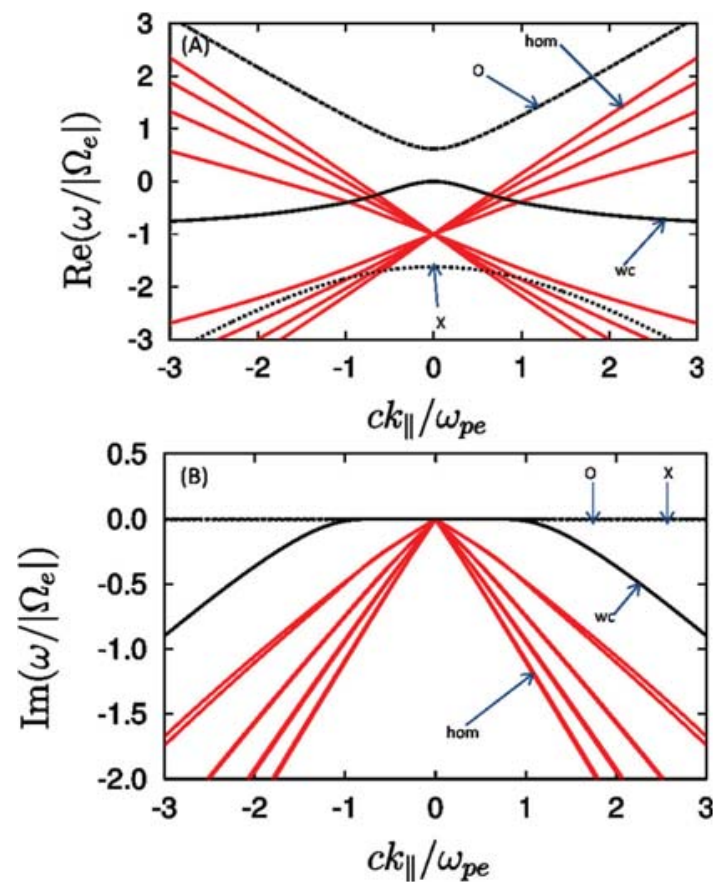

FIG. 2. Similar as in Fig. 1 but with $\beta_{e}=0.1$. 

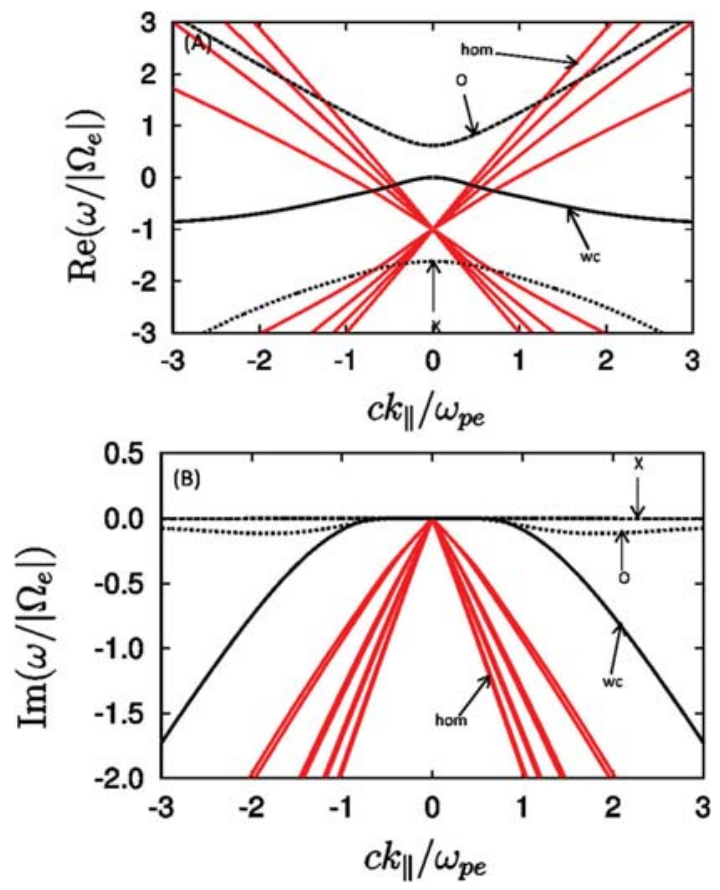

FIG. 3. Same as in Fig. 1 but with $\beta_{e}=0.3$.

electromagnetic waves in a stable thermal equilibrium plasma. Our aim in this section is to compute the spectrum of these fluctuations, first when the plasma is represented by a Maxwellian thermal distributions and later for a more general case of a nonthermal distribution modeled by a Tsallis-kappa-like distribution function. To describe the state of the plasma, we shall use the statistical ensemble average of the magnetic field through their deviations from the mean values (i.e., fluctuations) which are governed by the state of the plasma. The mean value of the fluctuations are equal to zero by definition; so the fluctuations are described by means of correlation functions (i.e., non-linear second order correlators) in the $\omega-\mathbf{k}$ Fourier domain. These are average products of fluctuations of different wavelengths at different frequencies. The averaging procedure is carried out over the mechanical or electrical state of the system and over the statistical distribution of various states represented by the correlation function. If the plasma medium is spatially homogeneous and only stationary states of the system are involved in the correlation, then the quadratic wavevector-frequency correlation function depends only on the relative distance and on the absolute value of the time segment between the points at which the fluctuations are examined. ${ }^{17}$

Following standard second-order methods, the classical spectral distribution of electric field fluctuations in a magnetized isothermal, and homogeneous plasma can be generally expressed (see Sitenko ${ }^{17}$ ) in terms of the fluctuationdissipation theorem where $\Lambda_{i j}^{-1}$ are the components of the inverse of the dispersion tensor [Eq. (1)], and $\langle\cdots\rangle$ represents an ensemble average

$$
\left\langle\delta E_{i} \delta E_{j}^{*}\right\rangle_{(\omega, \vec{k})}=4 \pi i \frac{k_{B} T}{\omega}\left(\Lambda_{i j}^{-1 *}-\Lambda_{j i}^{-1}\right) .
$$

For parallel propagation and using the Maxwell-Faraday equation, we can express this equation in terms of the magnetic fluctuations as

$$
\left\langle\left|\delta B_{ \pm}\right|^{2}\right\rangle_{\left(\omega, k_{\|}\right)}=8 \pi k_{B} T \frac{c^{2} k_{\|}^{2} \operatorname{Im}\left(\Lambda_{ \pm}^{*}\right)}{\omega^{3}} \frac{\left.\Lambda_{ \pm}\right|^{2}}{\mid x^{2}}
$$

whereas $\left\langle\left|\delta B_{\|}\right|^{2}\right\rangle=0$, and $\Lambda_{ \pm}$is given by Eq. (2) above.

Equation (9) has been solved numerically for the spectrum of magnetic fluctuations as a function of wave vector and frequency. The results of this calculation are illustrated

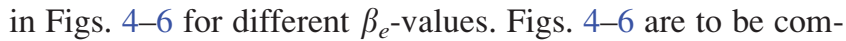
pared with the dispersion counterparts presented earlier in Figs. 1-3. Fig. 4 displays the contours and intensity scale of the magnetic fluctuations obtained from Eq. (9) for the same parameters as in Fig. 1. Also shown are the normal mode solutions calculated from the dispersion relation $\Lambda_{ \pm}=0$, representing the whistler-cyclotron, the extraordinary, and the ordinary wave normal modes of the system. The color scale shows the normalized power levels in logarithmic scale between $10^{-5}$ and $10^{-2}$. Comparing Figs. 4 and 1, it is clear that the spontaneous thermal fluctuations emerge in the triangle-like zones, i.e., they are constrained by the least damped higher-order modes (dashed lines), which are also solutions of the dispersion relation, indicating the zones bounding (limiting) the fluctuations of the whistler-cyclotron branch. Within these zones, the whistler-cyclotron, although still distinguishable from the background fluctuations, is heavily damped beyond $k_{\|} \lambda_{a} \geq 1.5$ as indicated in Fig. 1(b). In addition, Figs. 5 and 6 show the magnetic fluctuations for the cases with $\beta_{e}=0.1$ and $\beta_{e}=0.3$, respectively. As $\beta_{e}$ increases and the damped zones become broader, the magnetic fluctuations fill the extended regions at frequencies even larger than the cyclotron frequency, but otherwise with decreasing power. Most of the power is localized along the dispersion branch of the whistler-cyclotron mode, where the collective properties of the plasma reinforce the spontaneous fluctuations, i.e., the individual behavior of electrons. The power around the other two eigenmodes, ordinary and extraordinary waves, is also enhanced but at a significantly lower lever than for the whistler-cyclotron waves. This could be

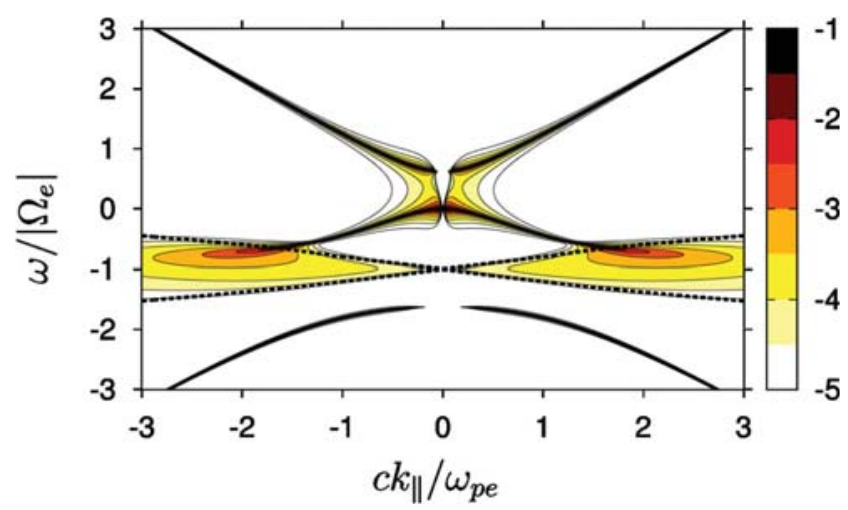

FIG. 4. Contours plot and intensity scale of the magnetic fluctuations obtained from Eq. (9) for a bi-Maxwellian plasma with the similar parameters as in Fig. 1. Dotted lines superposed are the least damped higher-order modes that confine the magnetic fluctuations. 


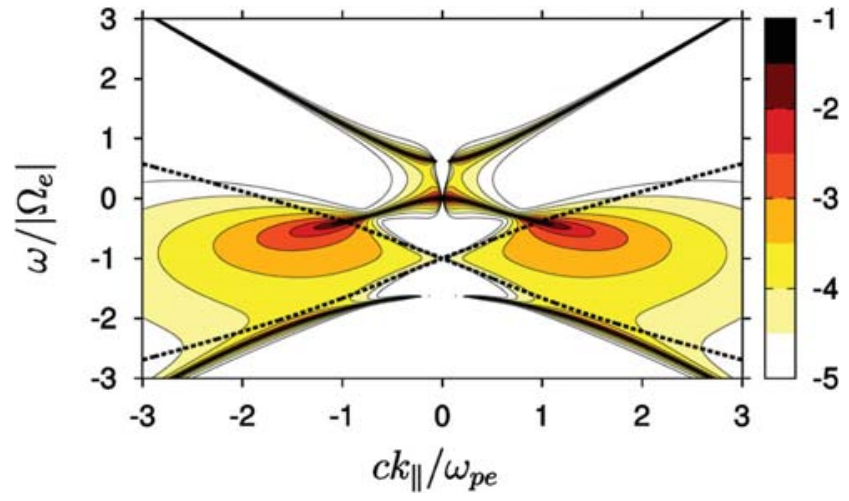

FIG. 5. Similar to Fig. 4 but with $\beta_{e}=0.1$.

due to the fact that we use a non-relativistic treatment of the fluctuations, and it could be possible that in the relativistic case, the $\mathrm{O}$ and $\mathrm{X}$ modes, which are mostly fast waves (phase speed higher than the speed of light in vacuo), should be able to resonate with the electrons. Note that, except for the symmetry breaking effects of temperature anisotropy or differential streaming due to collective effects, the fluctuation power is symmetric around the cyclotron frequency $-\left|\Omega_{e}\right|$ in a stable thermal equilibrium plasma. Therefore, for a Maxwellian plasma, the least damped higher-order modes provides a confining range of whistler-cyclotron fluctuations of the system. These results also shows that as $\beta_{e}$ increases the point where the whistler-cyclotron waves intersect the electromagnetic fluctuations shift towards longer wavelengths (i.e., smaller $k_{\|} \lambda_{e}$ ). This intersection region is interpreted as the location where the whistler-cyclotron waves begin to loose their identity by merging with the fluctuations in the plasma, which are heavily damped.

\section{A. Suprathermal effects}

Here, we consider the topology of the spectrum of magnetic fluctuations for a more general case of a nonthermal distribution modeled by a Tsallis-kappa-like distribution function. ${ }^{21-23}$ It is assumed that the plasma is composed of parallel drifting electrons and protons that obey the Tsallis-kappa-like particle velocity distribution function given by

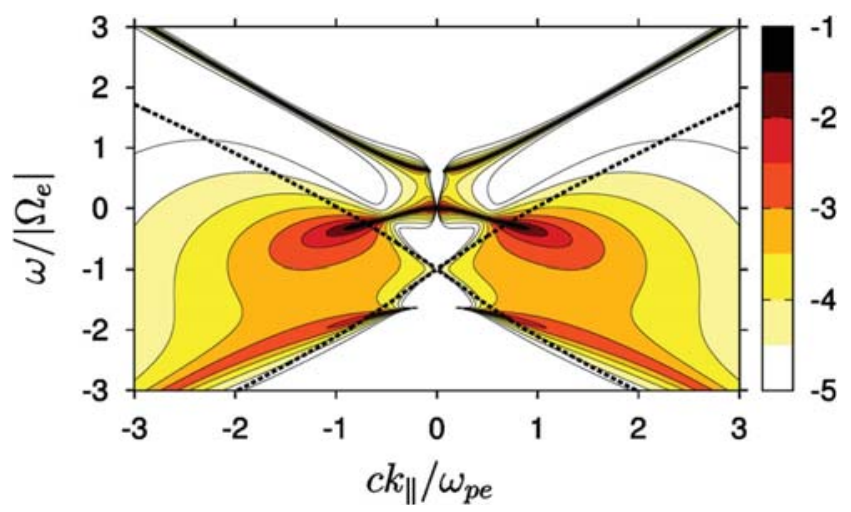

FIG. 6. Same as Fig. 4 but with $\beta_{e}=0.3$.

$$
\begin{aligned}
F_{s \kappa}\left(v_{\|}, v_{\perp}\right) & =A_{s \kappa}\left[1+\frac{1}{\kappa}\left(\frac{\left(v_{\|}-U_{\| s}\right)^{2}}{\alpha_{\| s}^{2}}+\frac{v_{\perp}^{2}}{\alpha_{\perp s}^{2}}\right)\right]^{-(\kappa+1)}, \\
A_{s \kappa} & =\frac{1}{\pi^{3 / 2} \alpha_{\| s} \alpha_{\perp s}^{2}} \frac{\Gamma(\kappa+1)}{\kappa^{3 / 2} \Gamma(\kappa-1 / 2)} .
\end{aligned}
$$

Here, the parameter $\kappa$ represents the degree on non-thermal effects. When $\kappa$ is small, the velocity distribution in Eq. (10) represents an effectively hotter distribution than a Maxwellian, whereas for $\kappa$ large and approaching infinity, the effective temperature reduces to that of a Maxwellian distribution. This effective temperature $T_{\kappa}$ of a Tsalliskappa-like distribution can be defined in terms of the Maxwellian temperature $T$ by obtaining the second moment of the distribution function Eq. (10) to yield

$$
T_{\kappa}=\frac{\kappa}{\kappa-3 / 2} T \text {. }
$$

Substituting Eq. (10) into Eqs. (2)-(4), we can write the perpendicular dispersion tensor as follows:

$$
\begin{aligned}
\Lambda_{ \pm}\left(\omega, k_{\|}\right)= & 1-\frac{k_{\|}^{2} c^{2}}{\omega^{2}}+\sum_{s=p, e} \frac{\omega_{p s}^{2}}{\omega^{2}} \\
& \times\left[\xi_{s} Z_{\kappa}\left(\xi_{s}^{ \pm}\right)+\Gamma_{s}\left(1+\xi_{s}^{ \pm} Z_{\kappa}\left(\xi_{s}^{ \pm}\right)\right)\right], \\
Z_{\kappa}(\xi)= & \frac{\Gamma(\kappa)}{\pi^{1 / 2} \kappa^{1 / 2} \Gamma(\kappa-1 / 2)} \\
& \times \int_{-\infty}^{+\infty} \frac{d t}{(t-\xi)}\left[1+\frac{t^{2}}{\kappa}\right]^{-\kappa}, \quad \operatorname{Im}(\xi)>0,
\end{aligned}
$$

where, for $\kappa>1 / 2$, the function $Z_{\kappa}(\xi)$ can be written in terms of the Gauss hypergeometric function ${ }_{2} F_{1}$

$$
Z_{\kappa}(\xi)=i \frac{\kappa-1 / 2}{\kappa^{3 / 2}}{ }_{2} F_{1}\left[1,2 \kappa ; \kappa+1 ; \frac{1}{2}\left(1-\frac{\xi}{i \sqrt{\kappa}}\right)\right],
$$

and the arguments $\xi_{s}$ and $\xi_{s}^{ \pm}$have been defined in Eq. (6) above. Here, $Z_{\kappa}(\xi)$ is the analogous plasma dispersion function for a kappa-like distribution as defined by Mace and Hellberg $^{31}$ and Mace and Sydora. ${ }^{32}$

We now present an estimate of the power spectrum of magnetic fluctuations as defined by Sitenko, ${ }^{17}$ but solving the dispersion relation for the Tsallis-kappa-like velocity distribution in Eq. (10), using Eqs. (9) and (12). Fig. 7 represents the numerical results of the transverse dispersion relation $\left(\Lambda_{ \pm}=0\right)$ for a warm plasma composed of a nondrifting (i.e., $U_{\| s}=0$ ) isotropic Tsallis-kappa-like velocity distribution of ions and electrons $\left(T_{\perp \mathrm{s}} / T_{\| \mathrm{s}}=1\right)$, with $\beta_{e}=\beta_{p}=0.1$ and $\omega_{p e} /\left|\Omega_{e}\right|=1$, which are similar parameters as those used in Fig. 2 for a bi-Maxwellian plasma. Here we use a value of $\kappa=3$ that represents a particle velocity distribution with a substantial suprathermal tail. For comparison purposes, here, we show the same range of wave vectors $-3 \leq k_{\|} \lambda_{e} \leq 3$ and normalized frequency $-3 \leq \operatorname{Re}(\omega) /$ $\left|\Omega_{\mathrm{e}}\right| \leq 3$ shown in Fig. 2. The solid curves of Fig. 7(a) crossing the origin of the $(\operatorname{Re}(\omega), k)$-plane represent the standard 

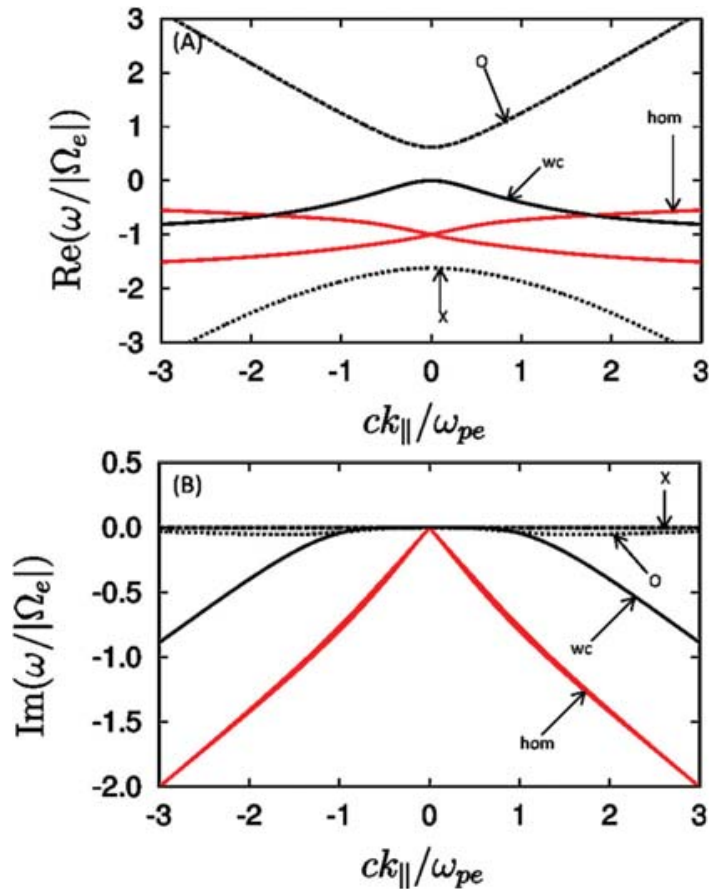

FIG. 7. (a) Real part of the solution of the transverse dispersion relation $\Lambda_{ \pm}=0$ for an isotropic electron-proton plasma following nonthermal distribution modeled by a Tsallis-kappa-like distribution function with $\kappa=3, m_{p} / m_{e}=1836, \omega_{p e} / \Omega_{e}=1$, and $\beta_{e}=0.1$. Solid curves correspond to whistler (wc), ordinary $(\mathrm{O})$ and extraordinary $(\mathrm{X})$ normal modes, and dotted lines are the hom. (b) Corresponding imaginary parts of wave modes shown in panel (a).

forward (third quadrant) and backward (fourth quadrant) propagating right-handed whistler-cyclotron modes which approaches resonance at the electron cyclotron frequency $-\left|\Omega_{e}\right|$. The other two wave modes correspond to the lefthanded ordinary and the right-handed extraordinary wave modes. Both modes approach asymptotically the speed of light at higher $k$-values. In Fig. 7(a), the ordinary left-handed wave modes are the black-dashed curves that hits the cut-off frequency at $k_{\|} \lambda_{e}=0$ just below the electron cyclotron frequency $\left(\omega \lesssim\left|\Omega_{e}\right|\right)$, whereas the extraordinary righthanded waves are the black-dashed curves that hits the cutoff frequency at $k_{\|} \lambda_{e}=0$ above the electron cyclotron frequency $\left(\omega \gtrsim-\left|\Omega_{e}\right|\right)$, similar to Fig. 2(a) above. These waves have their corresponding forward and backward propagation counterparts as shown on the other quadrants. Fig. 7(a) also includes two more solutions of the dispersion relation, corresponding to the least damped higher-order modes crossing at $\operatorname{Re}\left(\omega / \Omega_{\mathrm{e}}\right)=-1$ at $k_{\|} \lambda_{e}=0$ [red solid lines in Fig. 7(a)]. Although Figs. 7 and 2 have many similarities, one striking feature between the two solutions is that the number of higher-order modes is finite for a Tsallis-kappa-like distribution. Furthermore, the total number of higher-order modes $n$ obeys the relationship $n=(\kappa-1)$. Thus, for the case depicted in Fig. 7 where $\kappa=3$, the total number of higherorder modes is four. The higher-order modes in Fig. 7(a) are grouped very close together due to the rather effective higher temperature of the non-thermal distribution compared to the Maxwellian case. The $Z_{\kappa}$-function used in our calculations is essentially the same one defined by Hellberg and
Mace $^{33}$ in their Eq. (10). Using a similar treatment as Summers and Thorne, ${ }^{34}$ it can be shown that the dispersion relation is a polynomial of order $\kappa+2$ when $\kappa$ is integer and thus admits $\kappa+2$ complex solutions. After removing the electromagnetic modes $\mathrm{O}, \mathrm{X}$, and whistler, then the net number of higher-order modes reduces to $\kappa-1$. Alternatively, it can be shown that the ${ }_{2} F_{1}$ hypergeometric function for $\kappa$ integer is represented by a finite-order polynomial, whereas for $\kappa$ real, it has branch-cuts with infinite solutions.

Fig. 7(b) shows the damping rate of the main branches of the normal mode solutions and of the higher-order modes. For the whistler-cyclotron branch, the damping is nearly zero at long wavelengths and then increases substantially as the wave vector increases. For the ordinary branch, the damping is greater that for the Maxwellian case. This is because for the Tsallis-kappa-like distribution the effective temperature for $\kappa=3$ is higher than the Maxwellian, and therefore such cases are quite similar to a higher temperature Maxwellian case. For the extraordinary modes, the damping rate remains essentially zero. The damping of the higherorder modes increases in the directions given by the redsolid lines in Fig. 7(b) but the triangle-like zones formed by these modes in the $(\operatorname{Im}(\omega), k)$-plane show smaller dispersion among themselves than in the Maxwellian situation for the same $\beta_{e}=0.1$. However, they seem to be similar to the higher- $\beta_{e}$ case of the Maxwellian distribution [depicted in Fig. 3(b)].

In addition, Fig. 8 shows the corresponding electromagnetic fluctuations for the case of $\beta_{e}=0.1$ of a Tsallis-kappalike velocity distribution function with $\kappa=3$. As before, it shows clearly the presence of the whistler-cyclotron, extraordinary and ordinary normal modes of the system. The color scale shows logarithmic normalized power levels between $10^{-5}$ and $10^{-2}$. Note that in this case, the electromagnetic fluctuations are not bounded anymore by the least-damped higher order modes. This is because the effective temperature of this plasma [given by Eq. (11)] is effectively higher than a Maxwellian, i.e., $T_{\kappa}=2 T$ for such $k$-value. This is expected since the Tsallis-kappa-like distribution is a non-thermal distribution with an excess of suprathermal particles providing such effective higher temperatures. Thus, the electromagnetic

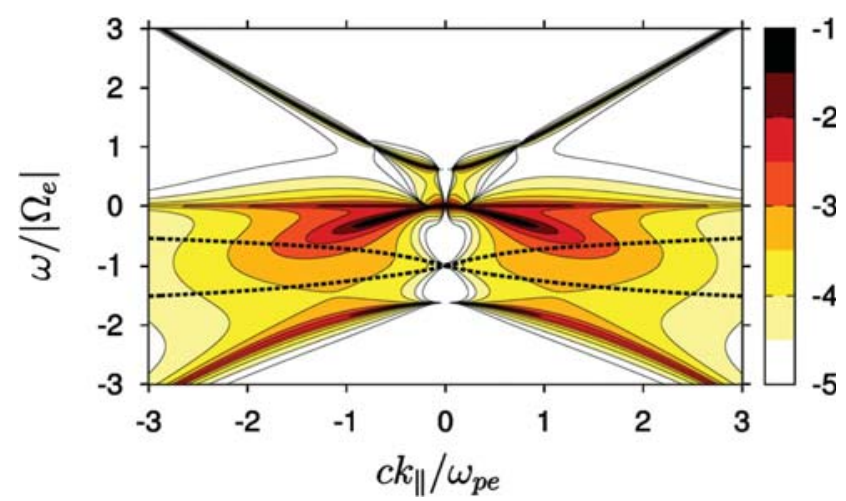

FIG. 8. Contours plots and intensity scale of the magnetic fluctuations obtained from Eq. (9) for a Tsallis-kappa-like plasma $(\kappa=3)$ with the same parameters as in Fig. 7 including the least damped higher-order modes (dashed lines). 
fluctuations can grow outside the limiting region of the least damped higher-order modes. Although not shown in here, this is also valid for different values of $\beta_{e}$ and $\kappa$. Thus, in summary, the higher order modes do not limit the electromagnetic fluctuations in a non-thermal plasma. This signature can be used as a diagnostic for detecting the presence of non-thermal plasmas in plasma systems where we have access to magnetic fluctuation data but we do not have direct in-situ measurements of the distribution function.

\section{FULL PARTICLE SIMULATIONS}

We now compare our theoretical calculations against full particle simulations for the same set of parameters used in Secs. I-III. We carry out particle-in-cell (PIC) simulations where the ions and electrons are treated kinetically and self self-consistently with the electric and magnetic field fluctuations. The simulation code is analogous to the KEMPO1 code of Omura and Matsumoto ${ }^{35}$ except for the schemes to correct the longitudinal electric field, the interpolation to the particle position and velocities, and the normalization. The simulation code is initiated with a Maxwellian distribution with a density and temperature consistent to the parameters used in Figs. 1-3. Periodic boundary conditions are imposed. The equations for the particle position and velocities are solved using the Boris-Buneman scheme and evolved in time by means of the leapfrog method. Moment charge densities and currents are calculated by a second-order Gaussian-like shape function by interpolation to the particle positions and velocities followed by accumulation of their charge and current contributions for each cell. This approach reduced considerably the noise level to obtain cleaner and smoothed results. Maxwell's equations for the electric and magnetic fields are solved via a second-order finite difference scheme. The longitudinal electric-field is corrected according to the Marder ${ }^{36}$ and Langdon ${ }^{37}$ schemes to assure that the longitudinal electric field determined via the Ampere's equation is self-consistent with that determined from Poisson's equation throughout the simulation. Time is normalized in units of the electron cyclotron frequency, particle positions are normalized to the electron inertial lengths, whereas all velocities are normalized to the speedof-light. Because the dimensional system of equations was written in cgs-units, the electric and magnetic fields were normalized similarly.

The temporal collisionless cyclotron damping of whistler waves has been studied by Scarf, ${ }^{38}$ Ossakow et al., ${ }^{39}$ Gitomer, ${ }^{18}$ and Gitomer and Forslund ${ }^{19}$ using numerical simulations. The calculated numerical dispersion of these modes agrees well with the prediction of a finite temperature linear dispersion theory. In our 1.5-D (i.e., one spatial and 3 velocity dimensions) simulations, the plasma is presumed to be collisionless, homogeneous, and magnetized, and it consists of a single ion and electron components with realistic mass ratio $m_{p} / m_{e}=1836$. Initially, electrons and protons satisfy an isotropic Maxwellian distribution, so that no free energy is present and the plasma is stable. To avoid side effects such as multi beam formation at low $\beta_{e}$, no quiet start particle-loading scheme was employed. The number of grid cells is 1024 with 1000 particles per species (electrons and protons) per cell. Similar results are obtained if 1600 particles per cell are used. The length of the simulation box is 512 in unit of the electron inertial length $\lambda_{e}$, the time step is $\Omega_{e} \Delta t=0.05$, and periodic boundary conditions were imposed for particles and fields. In this section, we focused only on the simulation results generated by a Maxwellian velocity distribution. The simulation results for an initial Tsallis-kappa like particle distribution will be presented later in a subsequent paper.

Figs. 9-11 represent the simulation counterparts of the theoretical results presented earlier in Figs. 4-6. Fig. 9 displays the $\omega-k$ power spectrum of the magnetic field fluctuations for $\beta_{e}=0.01$, obtained by Fourier-transforming the transverse magnetic field data both in space and time. The color scales show the power levels (normalized to the maximum wave power) in logarithmic scale between $10^{-6}$ and $10^{-2}$. After decomposing the wave fluctuations into positiveand negative-helicity parts, we can clearly recognize the forward (backward) propagating right-hand modes in the third (fourth) quadrants, respectively. Fig. 9 also shows details of the spontaneously emitted magnetic fluctuations, in agreement with Figs. 1 and 4. Note the triangle-shape zone in Fig. 9 beginning at $\omega /\left|\Omega_{e}\right|=-1$ near $\left|k_{||} \lambda_{e}\right|=0$ that encompass the whistler-cyclotron branch beyond $k_{\|} \lambda_{e} \geq 1$. In these triangle-shape zones, the whistler-cyclotron branch merges with the fluctuations, loosing its identity and becomes heavily damped. Figs. 10 and 11 depict the situations for the cases with $\beta_{e}=0.1$ and $\beta_{e}=0.3$, respectively, and illustrate that this merging region of the whistler-cyclotron waves and the fluctuations shift to lower $k_{\|} \lambda_{e}$ values as $\beta_{e}$ increases. Clearly, for the $\beta_{e^{-v a l u e s}}$ investigated, the spontaneous magnetic fluctuations are enhanced in the vicinity of the whistler-cyclotron modes, which yet quickly lose their identity at large values of $k_{\|} \lambda_{e}$ with increasing $\beta_{e}$. Thus, if enough free energy is available to overcome the natural threshold imposed by the thermal fluctuation level, the growth of linear and even nonlinear instabilities could be understood in terms of the amplification of some of the fluctuation modes. A quick comparison of Figs. 9 to 11 with Figs. 1 to 3 shows that although we have chosen the positive helicity solutions in the simulations, the results also includes

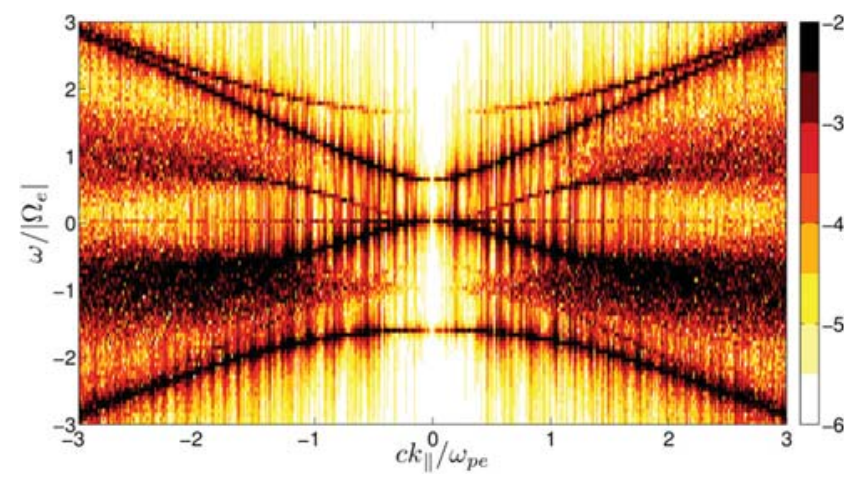

FIG. 9. Contour plot and intensity scale of the normalized magnetic power spectrum obtained from numerical particle-in-cell simulations of a electronproton Maxwellian plasma, with $m_{p} / m_{e}=1836, \omega_{p e} / \Omega_{e}=1$, and $\beta_{e}=0.1$ for an initial bi-Maxwellian plasma. 


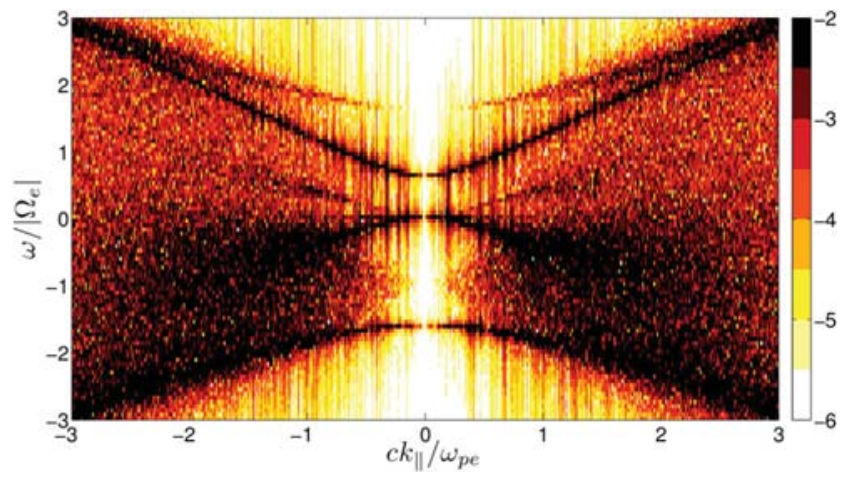

FIG. 10. Similar to Fig. 9 but with $\beta_{e}=0.1$.

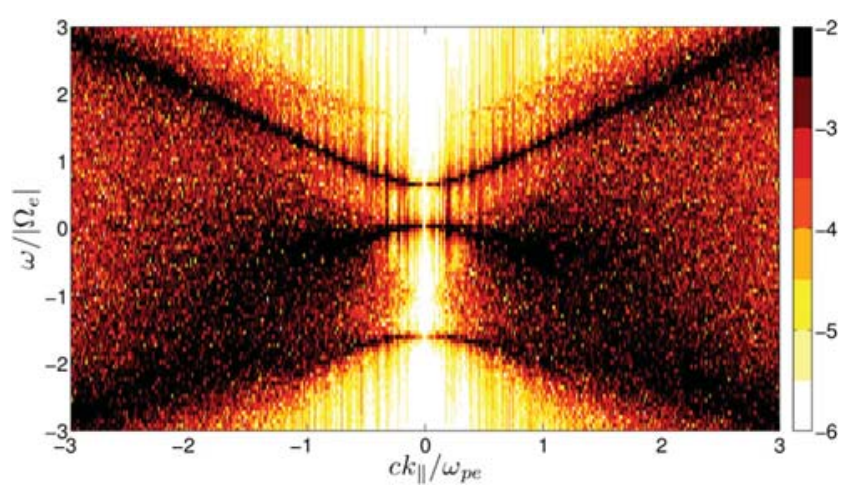

FIG. 11. Same as Fig. 9 but with $\beta_{e}=0.3$.

the mirror-image counter parts, corresponding to negative helicity, but at a much lower intensity. This is consistent with the mirror-image solutions obtained by changing the helicity sign in Figs. 1 to 3.

Fig. 12 shows a characteristic power spectrum obtained from the numerical PIC-simulations. The plot corresponds to a spatial Fourier mode $k_{\|} \lambda_{e}=0.075$ for the case of $\beta_{e}=0.01$ and $\omega_{p e} /\left|\Omega_{e}\right|=1$. The principal peak is the whistler cyclotron wave with a real frequency at $\omega /\left|\Omega_{e}\right|$ $=-0.31$. The lower amplitude higher frequency modes near $\omega /\left|\Omega_{e}\right|=-1.0$ are the fluctuations previously discussed. These results are consistent with the result plots obtained by

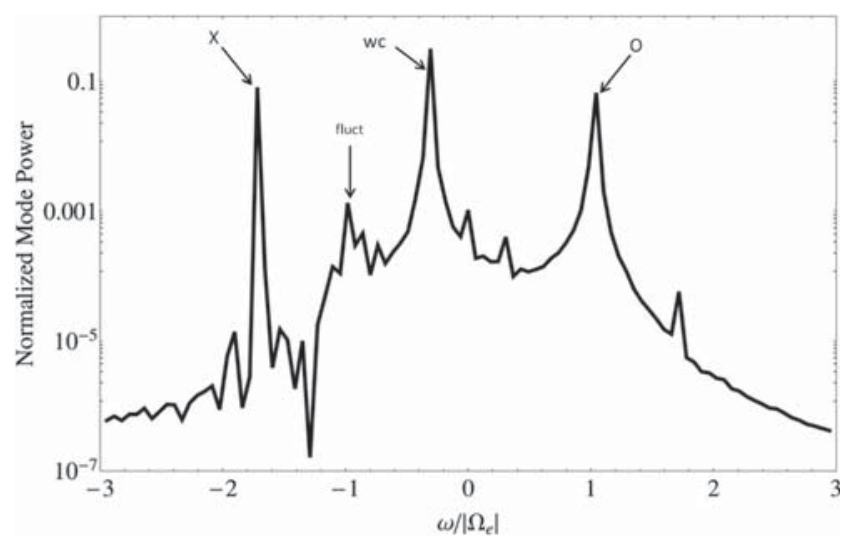

FIG. 12. Power spectrum versus normalized frequency for $k_{\|} \lambda_{e}=0.075$ and $\beta_{e}=0.01$ and $\omega_{p e} /\left|\Omega_{e}\right|=1$ displaying the principal peak of the whistler wave mode analogous to that of Gitomer and Forslund. ${ }^{19}$
Gitomer and Forslund ${ }^{19}$ (see their Fig. 3). There are two other peaks associated with the ordinary $(\mathrm{O})$ and extraordinary $(\mathrm{X})$ wave modes present in our simulation that do not appear in Gitomer and Forslung ${ }^{19}$ paper due to the fact that they use an implicit electromagnetic PIC-code that filter all the speed-of-light wave modes, whereas we have used and explicit PIC-code. Nonetheless, our results are consistently similar.

\section{CONCLUSIONS}

A description of the role of higher-order damped modes and their relation to the spontaneous magnetic fluctuations in magnetized thermal and nonthermal plasma has been presented. We have shown the relevance of higher-order modes in the emergence of spontaneous plasma fluctuations due to quasi-thermal noise through the fluctuation-dissipation theorem. From kinetic theory, the spectral distribution of the magnetic fluctuations is topologically constrained by the structure of the heavily damped higher order modes in a thermal plasma. These regions becomes broader as the plasma $\beta_{e}$ increases and they determine where instabilities could emerge if free energy is made available to the system. We also have shown that the zone where the whistler-cyclotron waves merges with the electromagnetic fluctuations shifts to longer wavelengths as $\beta_{e^{-i n c r e a s e s}}$ The zones where the whistler-cyclotron branch merges with the fluctuations are interpreted as regions where these waves lose their identity and becomes heavily damped. All our linear dispersion calculations and theoretical spectral fluctuation spectrum results exhibit remarkably consistent agreement with fully self-consistent numerical simulations for the same plasma conditions.

We have also carried out similar calculations for a nonthermal plasma modeled by a Tsallis-kappa-like velocity distribution function. In this case, the electromagnetic fluctuations are unconstrained by the higher-order modes because the Tsallis-kappa-like distribution has an excess of suprathermal particles that produce an effective temperature hotter than a thermal Maxwellian plasma. This result provides a critical observable signature, to determine the nature of a plasma, via its velocity distribution function, when using electromagnetic fluctuations data to diagnose the plasma even when direct in-situ measurements of the distribution function are not available. This important signature from kinetic theory should have applications to the understanding of the heating of electrons in laboratory and astrophysical plasmas. The calculations presented in this paper can be easily extended by analogy to the case of the low-frequency electromagnetic Alfven waves in which the ions play the major role. In summary, our results indicate that the spontaneously emitted electromagnetic fluctuations are in fact enhanced over these higher-order modes, suggesting that such modes play an important role in the emission and absorption of electromagnetic fluctuations in thermal or quasi-thermal plasmas.

\section{ACKNOWLEDGMENTS}

We would like to thank Dr. Robert Benson for providing critical suggestions and comments of this paper. We also like 
to thank the NASA's Wind/SWE program under the Grant No. NNX10AC56G for the support of this research. We also thank the Comisión Nacional de Ciencia y Tecnología (CONICyT, Chile) by providing financial support for postdoctoral (P.S.M.) and doctoral (R.N.) fellows. J.A.A. would like to thank FONDECYT (Nro 1110880) for providing financial support.

${ }^{1}$ J. C. Kasper, A. J. Lazarus, and S. P. Gary, Geophys. Res. Lett. 29, 1839, doi:10.1029/2002GL015128 (2002).

${ }^{2}$ E. Marsch, Living Rev. Sol. Phys. 3, 1 (2006).

${ }^{3}$ S. D. Bale, J. C. Kasper, G. G. Howes, E. Quataert, C. Salem, and D. Sundkvist, Phys. Rev. Lett. 103, 211101 (2009).

${ }^{4}$ P. H. Yoon, Phys. Plasmas 14, 064504 (2007).

${ }^{5}$ R. C. Tautz and R. Schlickeiser, Phys. Plasmas 14, 102102 (2007).

${ }^{6}$ R. Schlickeiser and P. H. Yoon, Phys. Plasmas 19, 022105 (2012).

${ }^{7}$ J. Araneda, H. Astudillo, and E. Marsch, Space. Sci. Rev. 172, 361 (2012).

${ }^{8}$ M. Lazar, P. H. Yoon, and R. Schlickeiser, Phys. Plasmas 19, 122108 (2012).

${ }^{9}$ T. Felten, R. Schlickeiser, P. H. Yoon, and M. Lazar, Phys. Plasmas 20, 052113 (2013).

${ }^{10}$ J. J. Seough and P. H. Yoon, Phys. Plasmas 16, 092103 (2009).

${ }^{11}$ P. H. Yoon, J. J. Seough, K. K. Khim et al., Phys. Plasmas 17, 082111 (2010).

${ }^{12}$ H. B. Callen and T. A. Welton, Phys. Rev. 83, 34 (1951).

${ }^{13} \mathrm{~N}$. Meyer-Vernet, P. Couturier, S. Hoang, J. L. Steinberg, and R. D. Zwickl, J. Geophys. Res. 91, 3294, doi:10.1029/JA091iA03p03294 (1986).

${ }^{14}$ E. J. Lund, J. LaBelle, and R. A. Treumann, J. Geophys. Res. 99, 23651, doi:10.1029/94JA02134 (1994).

${ }^{15} \mathrm{M}$. Moncuquet, A. Lecacheux, N. Meyer-Vernet, B. Cecconi, and W. Kurth, Geophys. Res. Lett. 32, L20S02, doi:10.1029/2005GL022508 (2005).

${ }^{16}$ G. Bekefi, Radiation Processes in Plasmas, Wiley Series in Plasma Physics (John Wiley and Sons, 1966), Chap. 4.
${ }^{17}$ A. Sitenko, Electromagnetic Fluctuations in Plasma (Academic Press, 1967).

${ }^{18}$ S. J. Gitomer, Phys. Fluids 16, 337 (1973).

${ }^{19}$ S. J. Gitomer and D. W. Forslund, Phys. Fluids 17, 1428 (1974).

${ }^{20}$ Y. Murata, I. Ogawa, and R. Sugaya, J. Phys. Soc. Jpn. 48, 1405 (1980).

${ }^{21}$ C. Tsallis, J. Stat. Phys. 52, 479 (1988).

${ }^{22} \mathrm{C}$. Tsallis and E. Brigatti, Continuum Mech. Thermodyn. 16, 223 (2004).

${ }^{23} \mathrm{C}$. Tsallis, Introduction to Nonextensive Statistical Mechanics: Approaching a Complex World (Springer Science and Business Media, LLC, 2009), Chaps. 2-3.

${ }^{24}$ H. Derfler and T. C. Simonen, Phys. Fluids 12, 269 (1969).

${ }^{25}$ S. P. Gary and R. L. Tokar, J. Geophys. Res. 90, 65, doi:10.1029/JA090iA01p00065 (1985).

${ }^{26}$ K. Matsuda, J. Plasma Phys. 36, 465 (1986).

${ }^{27}$ H. F. Astudillo, J. Geophys. Res. 101, 24433, doi:10.1029/96JA01586 (1996).

${ }^{28}$ S. P. Gary, Theory of Space Plasma Microinstabilities (Cambridge University Press, 1993).

${ }^{29}$ T. Stix, Waves in Plasmas (American Institute of Physics, 1992).

${ }^{30}$ B. D. Fried and S. D. Conte, The Plasma Dispersion Function (Academic, San Diego, California, 1961).

${ }^{31}$ R. L. Mace and M. A. Hellberg, J. Plasma Phys. 43, 239 (1990).

${ }^{32}$ R. L. Mace and R. D. Sydora, J. Geophys. Res. 115, A07206, doi:10.1029/2009JA015064 (2010)

${ }^{33}$ M. A. Hellberg and R. L. Mace, Phys. Plasmas 9, 1495 (2002).

${ }^{34}$ D. Summers and R. M. Thorne, Phys. Fluids 3, 1835 (1991).

${ }^{35} \mathrm{Y}$. Omura and H. Matsumoto, Computer Space Plasma Physics: Simulation Techniques and Software (Terra Scientific Publishing Company, Tokyo, 1993), Chap. 2.

${ }^{36}$ B. Marder, J. Comput. Phys. 68, 48 (1987).

${ }^{37}$ A. B. Langdon, Comput. Phys. Commun. 70, 447 (1992).

${ }^{38}$ F. L. Scarf, J. Geophys. Res. 67, 1751, doi:10.1029/JZ067i005p01751 (1962).

${ }^{39}$ S. L. Ossakow, I. Haber, and R. N. Sudan, Phys. Fluids 15, 935 (1972). 